\title{
Diagnóstico prenatal y evolución en fetos con agenesia de ducfus venoso
}

\author{
Javier Pérez-Pedregosa ${ }^{1,4}$, María Teresa Martínez G. ${ }^{1}$, Sandra del Pino S. R. ${ }^{1}$, Federico \\ Gutiérrez-Larraya A. ${ }^{2}$, Leticia Medina M. ${ }^{3}$, Teresa Bueso M. ${ }^{1}$, Eduardo Cabrillo R. ${ }^{1,4}$ \\ ${ }^{1}$ Departamento de Medicina Materno Fetal, Servicio de Ginecología y Obstetricia, Hospital Sanitas La Moraleja, Ma- \\ drid. ${ }^{2}$ Servicio de Cardiología Pediátrica, Hospital Sanitas La Moraleja, Madrid. ${ }^{3}$ Departamento Diagnóstico Prenatal, \\ Servicio de Ginecología y Obstetricia, Hospital Sanitas La Zarzuela, Madrid. ${ }^{4}$ Universidad Francisco de Vitoria, Madrid, \\ España.
}

\section{RESUMEN}

Antecedentes: El ductus venoso es una derivación vascular (shunt) presente en el feto que permite el paso de sangre oxigenada de la vena umbilical (VU) hacia la circulación coronaria y cerebral. Su agenesia se asocia con defectos cromosómicos, síndromes genéticos, defectos estructurales y complicaciones prenatales como crecimiento intrauterino retardado y muerte fetal. Resultados: Se analizaron 15 agenesias de ductus venoso (ADV) en gestaciones únicas entre enero de 2010 y diciembre de 2013. El 80\% de ellas fueron diagnosticadas en la exploración rutinaria de la semana 12. Se realizó estudio de cariotipo en el $53 \%$ de los casos (8/15), bien por riesgo alto de cromosomopatía en el cribado combinado y/o translucencia nucal aumentada (75\%) o malformaciones asociadas (25\%). Sólo hubo un diagnóstico de trisomía 21 y postnatalmente de una microdelección del cromosoma 7. Realizaron interrupción legal del embarazo un total de 4 pacientes (por trisomía 21 o por alteraciones estructurales). Entre las 11 gestaciones restantes un $27 \%$ se diagnosticó RCIU, hubo una muerte neonatal a las 12 horas de vida por síndrome de aspiración meconial e hipertensión pulmonar. En un $60 \%$ se objetivó la presencia de un drenaje umbilicohepático y entre los 6 restantes con shunt portosistémico, 4 tenían conexión entre la VU y la VCl. Conclusión: La ADV es una anomalía infrecuente del sistema venoso fetal, de difícil diagnóstico y con mal pronóstico en aquellos casos en que se asocia con otros marcadores y/o anomalías estructurales que pueden aparecer tardíamente, por lo que debe realizarse un seguimiento adecuado.

\section{PALABRAS CLAVE: Agenesia de ductus venoso, ductus venoso, diagnóstico prenatal, ecografía 3D doppler color, sistema venoso fetal, sistema portal fetal, derivación vascular}

\section{SUMMARY}

Background: The ductus venosus (DV) is a unique shunt that allows direct passage of oxygenated blood from the umbilical vein (UV) to the coronary and cerebral circulation by a preferential passage through the foramen ovale. DV agenesis (DVA) is associated with chromosomal abnormalities, genetic syndromes, structural defects and prenatal complications such as intrauterine growth retardation (IUGR) or even stillbirth. Results: We report 15 cases of DVA in singleton pregnancies between January 2010 and December 2013. $80 \%$ of them were diagnosed on routine examination during the 11-14 weeks scan. Karyotyping was performed in $53 \%$ of cases (8/15) by high risk of chromosomal abnormalities in the first trimester combined screening and/or an increased nuchal translucency thickness in 6/8 (75\%), or associated malformations $2 / 8$ 
(25\%). There was only one fetus diagnosed of trisomy 21 by amniocentesis and another fetus was postnatally diagnosed of a microdeletion of chromosome 7. 4 patients performed legal abortions (the trisomy 21 and in 3 cases for severe structural malformations). Among the remaining 11 pregnancies, $3(27 \%)$ were diagnosed with IUGR and there was a neonatal death at 12 hours of life for meconium aspiration syndrome and pulmonary hypertension. $60 \%$ of the fetus presented an intrahepatic drainage and among the remaining 6 with portosystemic shunt, in 4 a connection between the UV and the inferior vena cava was observed. Conclusion: DVA is a rare anomaly of the fetal venous system, difficult diagnosis and poor prognosis in cases associated with other markers and/or structural abnormalities that may even appear late. A detailed survey of fetal anatomy and follow up of these fetuses is necessary.

\section{KEY WORDS: Ductus venosus agenesis, ductus venosus, prenatal diagnosis, 3D Doppler color ultra-} sound, fetal venous system, fetal portal system, vascular shunt

\section{INTRODUCCIÓN}

El ductus venoso fetal (DV) es un shunt único que permite el paso directo de sangre bien oxigenada desde la vena umbilical (VU) a la circulación coronaria y cerebral a través de un paso preferencial por el foramen oval. Se comporta como una vena arterializada con un flujo anterógrado de alta pulsatilidad durante todo el ciclo cardiaco (1-3) (Figura 1). Es habitual su estudio durante la exploración ecográfica de 11+0 - 13+6 semanas, bien de forma rutinaria o como marcador de segundo orden, ya que la presencia de flujo ausente o reverso durante la contracción atrial es un marcador de aneuploidías y/o cardiopatías.

La agenesia de este relevante shunt se ha asociado con anomalías cromosómicas y síndromes genéticos raros, defectos estructurales, RCIU e incluso muerte fetal anteparto (4-6). Es una anomalía rara y de difícil diagnóstico, aunque algunas series publicadas por grupos muy expertos y en población de alto riesgo lo describen en hasta 6/1000 exploraciones (7).

Dos rutas diferentes para el retorno de la VU han sido descritos en estos fetos; drenaje extrahepático o shunt portosistémico (conexión directa de la VU a la vena iliaca, a la cava inferior ( $\mathrm{VCl}$ ), a la aurícula derecha $(A D)$, a las venas suprahepáticas o incluso al seno coronario) (5-8) o drenaje intrahepático o shunt umbilico hepático (conexión de la VU al seno portal).

El objetivo de este trabajo es evaluar las características y evolución de los fetos valorados con diagnóstico de agenesia de ductus venoso (ADV) en nuestra Unidad de Diagnóstico Prenatal del Hospital Sanitas La Moraleja de Madrid durante un periodo de 4 años.

\section{PACIENTES Y MÉTODO}

Entre todas las gestaciones únicas valoradas en nuestra Unidad entre enero de 2010 y diciembre de
2013, hemos analizado aquellas en las que se confirmó la ADV aislada o asociada a otras anomalías morfológicas. El diagnóstico inicial se estableció con la demostración mediante Doppler color en modo B de la ausencia de conexión entre la $\mathrm{VU}$ y la $\mathrm{VCl}$ en los distintos planos para visualizar el flujo ductal. En todos los casos se obtuvieron volúmenes con ecografía tridimensional Doppler color para el estudio e identificación off- line de la localización del tipo de shunt, así como la grabación de las exploraciones en aquellos casos de mayor complejidad diagnóstica.

Se describe la edad gestacional al diagnóstico, la forma de presentación y el tipo de drenaje venoso encontrado, así como la presencia de anomalías asociadas y/o alteraciones del cariotipo fetal. Hemos seguido la evolución de la gestación y tras el nacimiento en aquellos casos en que la gestación continuó (Tabla I).

\section{RESULTADOS}

Durante el periodo de estudio, un total de $15 \mathrm{fe}$ tos fueron diagnosticados de ADV (Tabla I). En 12 $(80 \%)$ se diagnosticó o al menos se sospechó durante la exploración de 11+0 - 13+6 semanas, con un rango de 11 a 26 semanas. El hallazgo inicial en todos los casos fue la imposibilidad de estudio de la onda de velocidad de flujo (OVF) del DV tanto en el corte medio sagital similar al empleado para la medición de la translucencia nucal (TN) (Figura 2), como en un corte axial a nivel de la entrada de la vena umbilical en el abdomen fetal pese a situar la ventana de Doppler color o incluso Power Doppler de mayor sensibilidad, en dicha región identificando el resto de vascularización fetal. En aquellos casos de duda, utilizamos Doppler pulsado para distinguir el flujo de vasos vecinos como las venas suprahepáticas o incluso la VCl (Figura 1). 
Tabla I

ANTECEDENTES CLÍNICOS DE LA SERIE DE CASOS ANALIZADA

\begin{tabular}{|c|c|c|c|c|c|c|}
\hline Caso & $\begin{array}{c}\text { Edad } \\
\text { materna }\end{array}$ & $\begin{array}{c}\text { Edad } \\
\text { gestacional al } \\
\text { diagnóstico }\end{array}$ & $\begin{array}{l}\mathrm{TN} \\
(\mathrm{mm})\end{array}$ & $\begin{array}{l}\text { Cariotipo } \\
\text { fetal }\end{array}$ & Anomalías asociadas & Evolución neonatal \\
\hline 1 & 35 & $13+3$ & 2,8 & No & RCIU leve & RCIU, Vivo sano \\
\hline 2 & 37 & $13+0$ & 3,8 & Trisomía 21 & No & IVE \\
\hline 3 & 35 & $20+0$ & 3,9 & $46 X X$ & No & Vivo sano \\
\hline 4 & 23 & $12+2$ & 1,1 & No & $\begin{array}{l}\text { Polihidramnios } \\
\text { RCIU tipo I severo }\end{array}$ & $\begin{array}{c}\text { Parto pretérmino, RCIU } \\
\text { Síndrome } \\
\text { polimalformativo } \\
\text { Microdelección } \\
\text { cromosoma } 7\end{array}$ \\
\hline 5 & 30 & $26+1$ & 1,1 & $46 X X$ & $\begin{array}{c}\text { AUU, PVCSI } \\
\text { Agenesia parcial } \\
\text { sistema porta } \\
\text { Dominancia cavidades } \\
\text { derechas }\end{array}$ & $\begin{array}{c}\text { Muerte neonatal } \\
\text { SAM e HTP }\end{array}$ \\
\hline 6 & 43 & $12+0$ & 1,3 & $46 X Y$ & $\begin{array}{c}\text { Agenesia cuerpo } \\
\text { calloso }\end{array}$ & IVE \\
\hline 7 & 38 & $12+5$ & 8,9 & $46 X Y$ & PVCSI, RCIU severo & Parto pretérmino, RCIU \\
\hline 8 & 36 & $13+0$ & 2,8 & $46 x X$ & $\begin{array}{c}\text { AUU } \\
\text { Hipoplasia cuerpo } \\
\text { calloso y vermis } \\
\text { inferior }\end{array}$ & IVE \\
\hline 9 & 37 & $11+6$ & 1,1 & No & Derrame pericárdico & $\begin{array}{c}\text { Miocardiopatía } \\
\text { hipertrófica leve }\end{array}$ \\
\hline 10 & 37 & $20+0$ & 1,5 & No & $\begin{array}{c}\text { PVUD } \\
\text { Agenesia parcial } \\
\text { sistema porta }\end{array}$ & Vivo sano \\
\hline 11 & 35 & $13+2$ & 1,6 & No & No & Vivo sano \\
\hline 12 & 40 & $12+2$ & 15 & $46 X X$ & Higroma quístico & IVE \\
\hline 13 & 35 & $11+4$ & 2,8 & $46 X Y$ & No & Vivo sano \\
\hline 14 & 41 & $11+5$ & 1,0 & No & No & Vivo sano \\
\hline 15 & 27 & $12+4$ & 1,4 & No & AUU & Vivo sano \\
\hline
\end{tabular}

TN: translucencia nucal. RCIU: restricción del crecimiento intrauterino. AUU: arteria umbilical única. PVCSI: persistencia de vena cava superior izquierda. PVUD: persistencia de vena umbilical derecha. SAM: síndrome de aspiración meconial. IVE: interrupción voluntaria de embarazo. HTP: hipertensión pulmonar. 


\section{OVF Sistema Venoso Fetal}

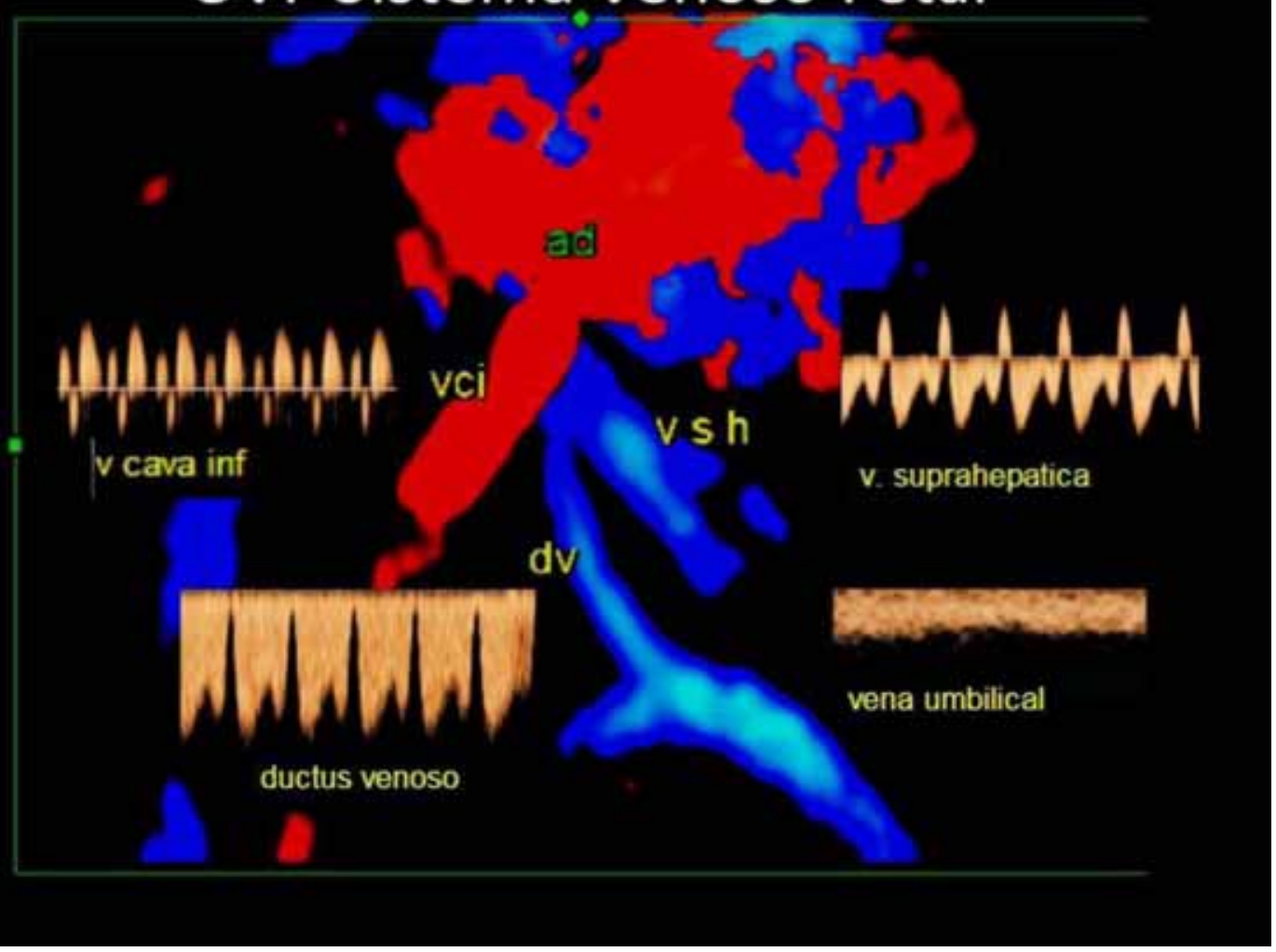

Figura 1. Reconstrucción 3D doppler color del sistema venoso fetal y representación de las ondas de flujo con doppler pulsado en la vena cava inferior (VCI), venas suprahepáticas (VSH), ductus venoso (DV), y vena umbilical (VU). El flujo a través del DV muestra un patrón trifásico característico con velocidades anterógradas durante todo el ciclo cardíaco.

En algo más de la mitad de los casos se realizó estudio del cariotipo fetal (8 de 15). En 6 de estos $8(75 \%)$, la indicación fue un índice de riesgo en el cribado combinado $\geq 1 / 100$ para alguna de las trisomías estudiadas. En el resto se realizaron por marcadores o anomalías asociadas; persistencia de vena cava superior izquierda (PVCSI) y arteria umbilical única (AUU) (caso 5) y agenesia de cuerpo calloso (ACC) (caso 6).

Uno de los fetos fue diagnosticado de trisomía 21. La otra alteración cromosómica fue diagnosticada postnatalmente en un feto que presentaba prenatalmente inquietantes e inespecíficos hallazgos asociados: AUU, RCIU, polihidramnios y rasgos faciales dismórficos. Tras el nacimiento a las 33 semanas de un varón de 1640 gramos, se confirmaron los rasgos dismórficos así como una microdelección del cromosoma 7, sin que hasta la fecha haya sido clasificado dentro de algún cuadro sindrómico específico. El resto presentaron un cariotipo normal.

Un total de 7 de los 15 fetos (46\%) presentaban una TN superior al percentil 95, pero sólo en 2 de ellos $(13 \%)$ el cariotipo fue anormal. Se diagnosticaron anomalías estructurales y/o marcadores vasculares asociados en 7 de 15 (46\%). 2 de 15 fueron diagnosticados en la mitad de la gestación de anomalías del sistema nervioso central (ACC aislada e hipoplasia del calloso y vermis inferior y AUU).

Tras el diagnóstico, un total de 4 pacientes optaron por interrumpir la gestación por presentar una aneuploidía (trisomía 21) o alteraciones estructurales (ACC, hipoplasia del CC y vermis inferior, e higroma quístico). 
Entre los 11 recién nacidos hubo $3(27 \%)$ con diagnóstico de retraso de crecimiento intrauterino (RCIU), siendo uno de ellos en el contexto de un cuadro sindrómico diagnosticado postnatalmente y asociado a microdelección del cromosoma 7.

Hubo una muerte neonatal a las 12 horas de vida por síndrome de aspiración meconial (SAM) e hipertensión pulmonar (HTP) en un feto con ADV asociada con AUU y PVCSI. Uno de los fetos que únicamente presentaba derrame pericárdico asociado a la ADV fue diagnosticado tras el nacimiento de miocardiopatía hipertrófica leve. En 9 de los 15 $(60 \%)$ se diagnosticó de un drenaje umbilicohepático y entre los 6 restantes con shunt portosistémico, 4 tenían conexión entre la VU y la VCl.

La evolución fue similar independientemente del tipo de drenaje encontrado en nuestra muestra. Aunque los 2 fetos con RCIU no sindrómico y en 3 de 6 con TN aumentada tenían un drenaje portosistémico.

\section{DISCUSIÓN}

El estudio de la OVF en el DV, pese a ser un marcador considerado de segundo orden, es práctica común en muchos centros durante la exploración de las 11+0 - 13+6 semanas, ya que no añade gran complejidad ni tiempo a la valoración, si el operador tiene el entrenamiento necesario. La imposibilidad de obtener la señal color y OVF característica trifásica con flujo anterógrado a lo largo del ciclo (Figuras 1 y 2), o el detectar una onda de flujo patológica (flujo diastólico ausente o reverso durante la contracción atrial) supone una indicación para realizar un estudio morfológico precoz, más aún en presencia de otros marcadores asociados.

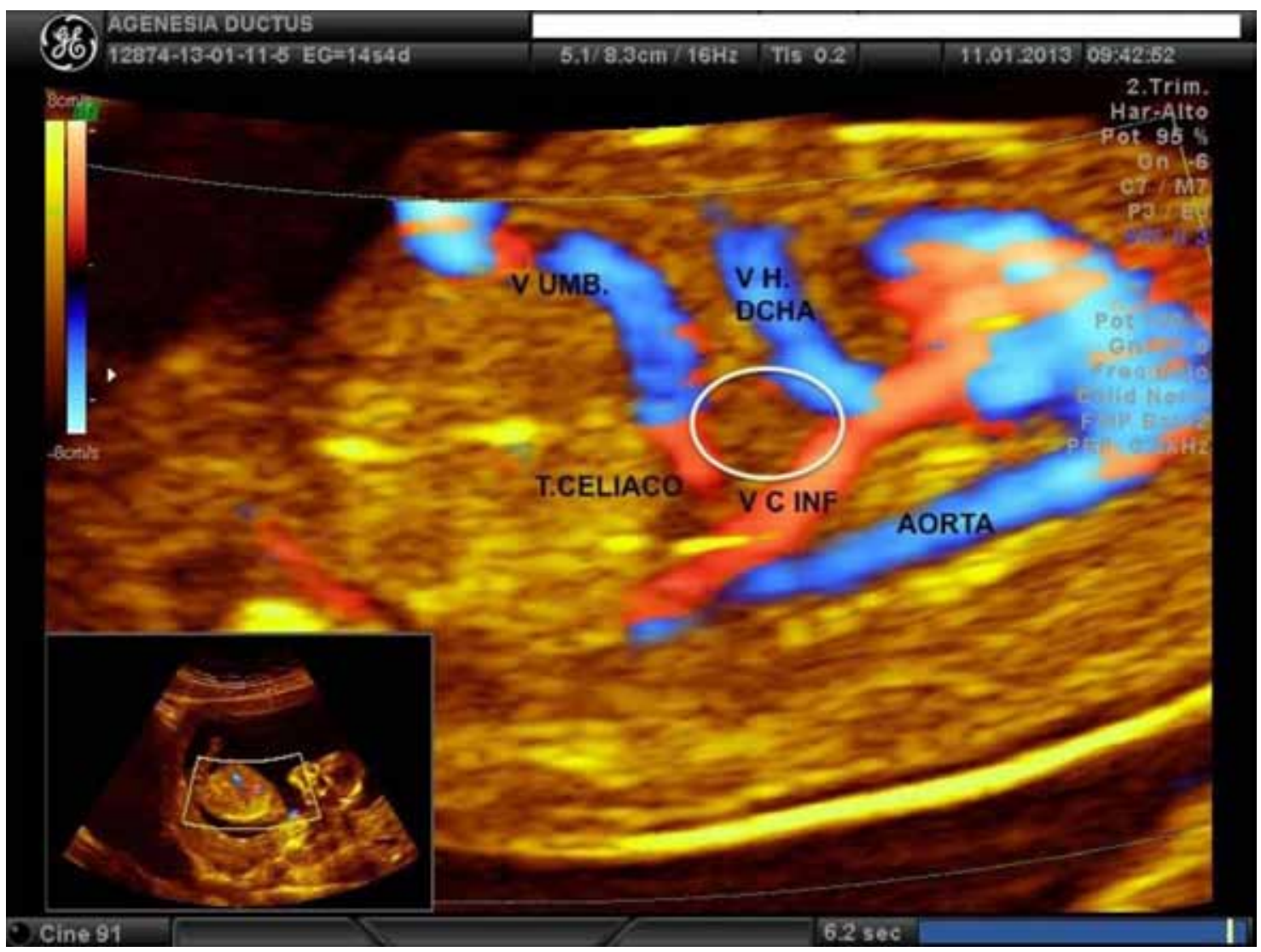

Figura 2. Corte medio sagital del abdomen fetal con Doppler color, donde no se consigue visualizar el ductus venoso, mientras que sí se identifica el resto de vascularización fetal. 
La ADV es consecuencia de un fallo en la formación de la anastomosis crítica entre el sistema umbilical y el vitelino, no existiendo comunicación directa entre la VU y el corazón derecho, y la sangre por tanto se desvía a través de un vaso aberrante bien hacia las venas extrahepáticas (shunt portosistémico) como la iliaca, vena cava inferior, vena cava superior o venas suprahepáticas. Bien hacia la $A D$ o directamente al seno coronario o a través de una red venosa intrahepática (shunt umbilico-hepático) siendo ésta la forma de presentación más frecuente (5). Se trata de una rara anomalía con una prevalencia de hasta 6/1000 exploraciones y una tasa de malformaciones asociadas muy variable (25-65\%), no sólo cardiopatías, sino de cualquier otra localización, así como aneuploidías y síndromes genéticos como el de Noonan (3-7).

El diagnóstico de ADV ha de ser seguido de una exhaustiva valoración morfológica y ecocardiográfica así como del sistema venoso fetal. En nuestro estudio encontramos hasta un $87 \%$ de anomalías y/o marcadores asociados (incluyendo alteraciones genéticas) la mayoría diagnosticados durante el estrecho seguimiento realizado en estos fetos. El pronóstico es desfavorable en presencia de otros hallazgos, es en esta situación cuando debe ofrecerse el estudio del cariotipo fetal.

Como hallazgo aislado se presenta en sólo el $35-60 \%$ de las ocasiones. En este escenario el pronóstico es marcadamente más favorable con un $80-100 \%$ de fetos con evolución absolutamente normal (5). Sólo en 2 de nuestros casos (13\%) la ADV fue aislada, aun así la evolución postnatal ha sido favorable en 9 de los 15 casos (60\%).

Una de las limitaciones de nuestro trabajo es que aquellos casos en los que se interrumpió la gestación no se realizó necropsia y en el neonato que falleció tras el parto por un SAM e HTP los padres renunciaron a su estudio posterior por lo que no se pudo descartar la presencia de otros hallazgos.

Hasta en el $50 \%$ de los casos se ha descrito su asociación con agenesia de la vena porta o incluso agenesia parcial o total del sistema porta. Shen y cols (8), señalan el desarrollo del sistema porta como el factor prenatal pronóstico más relevante en aquellos casos de agenesia aislada del DV. Si el shunt vascular es estrecho (Figura 3), similar al ductus, ayuda, según estos mismos autores, al normal desarrollo portal lo que tiene implicación favorable en la evolución postnatal. Un shunt grueso, del calibre de la umbilical, se asocia según estos mismos autores con peor evolución (Figura 4).

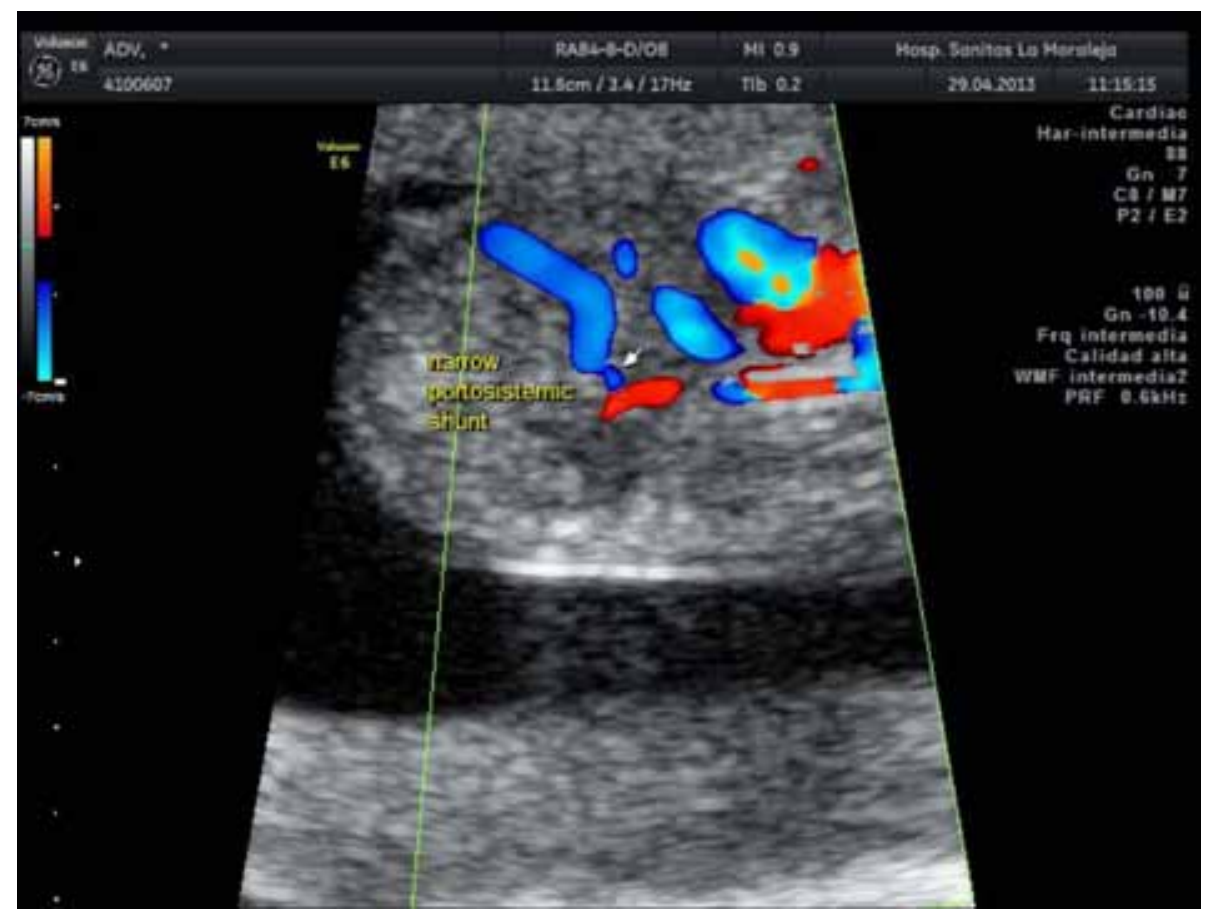

Figura 3. Corte medio sagital del abdomen fetal en un feto con ADV donde se visualiza un shunt portosistémico estrecho a vena cava inferior. 


\section{ADV SHUNT PORTOSISTEMICO A VCI}

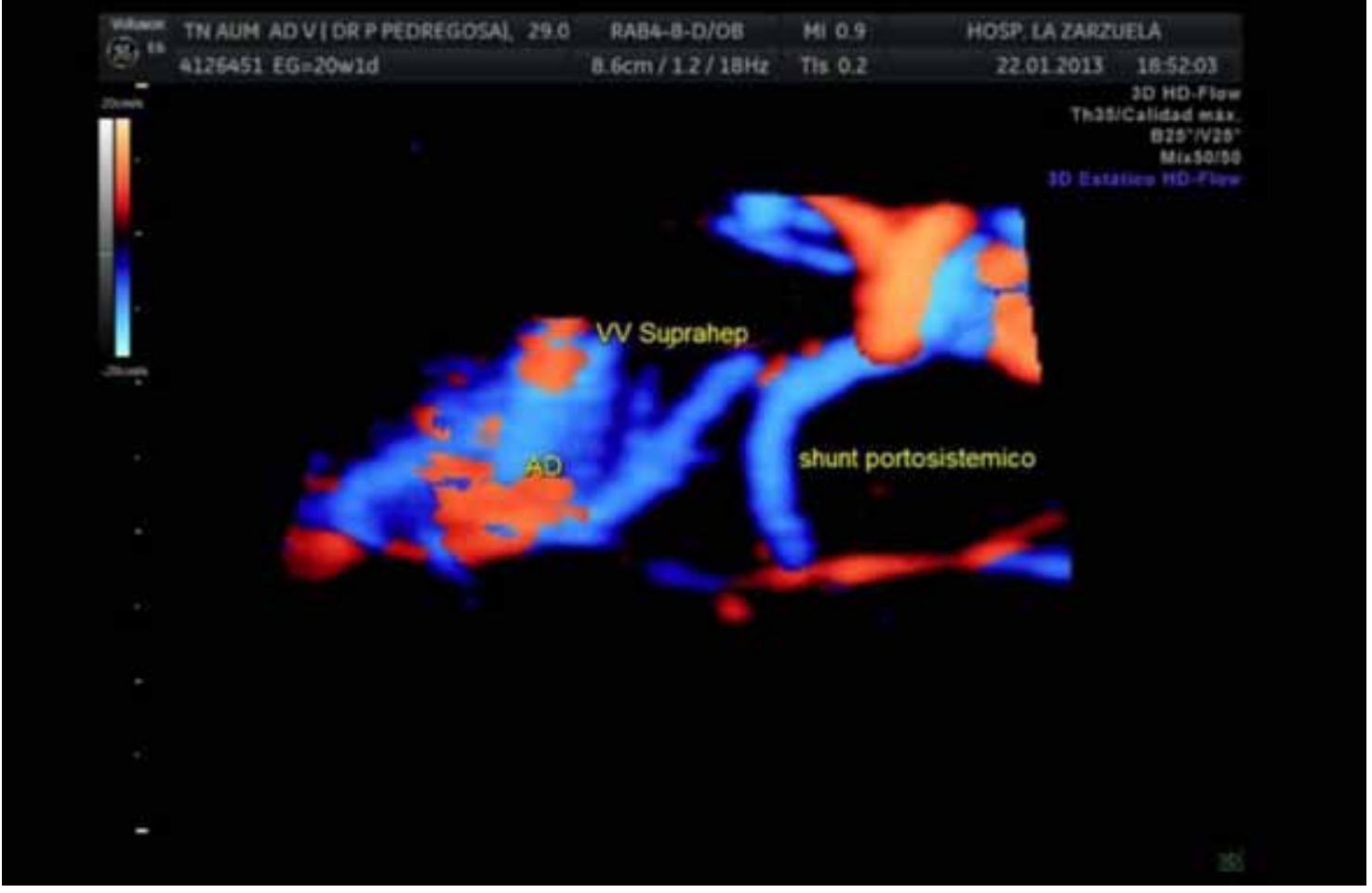

Figura 4. Reconstrucción tridimensional de la vascularización fetal con 3D Doppler HD en un feto con ADV donde se objetiva un grueso shunt portosistémico a vena cava inferior.

Para algunos autores, un factor pronóstico relevante es la localización del drenaje de la VU, ya que aquellos casos en los que no hay by pass hepático tienen mucha mejor evolución (7). Es importante reseñar la enorme dificultad que entraña en ocasiones demostrar la presencia de shunt portosistémico (Figura 5). Ante la no evidencia de drenaje extrahepático tras una exhaustiva búsqueda con Doppler color en modo B y 3D HD, objetivando el final del trayecto de la vena umbilical en el hígado y analizando meticulosamente el mapa color del sistema venoso portal establecemos el diagnostico de shunt umbilico-hepático.

En 23 casos de ADV Berg y cols (9), encontraron 15 aneuploidías y/o anomalías estructurales mayores. Entre los 8 restantes, sin graves anomalías asociadas, la evolución fue significativamente más favorable entre aquellos con drenaje intrahepático. Entre nuestros casos con drenaje extrahepático (shunt portosistémico), únicamente hemos encontrado una proporción mayor de fetos con TN aumentada y RCIU aunque la evolución fue similar. En nuestra experiencia el incremento del grosor de la nuca en esta población de fetos no se asocia con aumento del riesgo de aneuploidías cuando aparece de forma aislada y con marcadores bioquímicos de primer trimestre normales y podría estar ocasionada por alteraciones hemodinámicas transitorias por lo que no supone en nuestra opinión una clara indicación de estudio del cariotipo fetal.

La presencia de shunt extrahepático tiene dos consecuencias que se postulan como responsables del peor desenlace de estos fetos y neonatos. En primer lugar, la sobrecarga de volumen responsable de fallo cardiaco congestivo, y en segundo lugar, y no menos relevante, el posible hipodesarrollo hepático por el robo de volumen durante la vida fetal, más aún si la anomalía se asocia con alteración del sistema porta. 


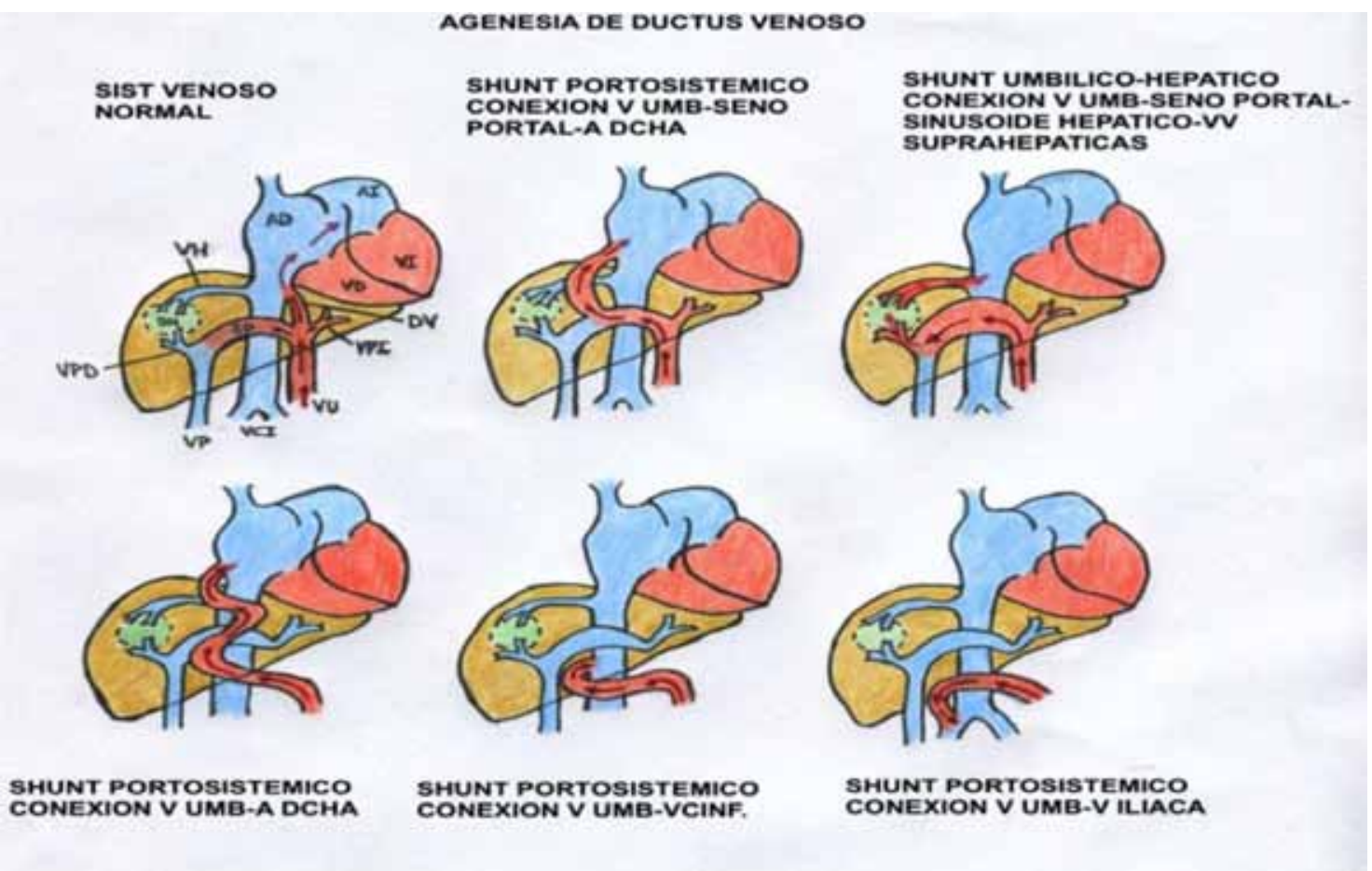

Figura 5. Esquema del sistema venoso fetal normal y los posibles shunts en fetos con agenesia del ductus venoso.

Este mismo grupo describió 19 casos con drenaje intrahepático sin anomalías mayores asociadas con supervivencia en el $100 \%$ de los casos, mientras que sólo 20 de los 29 casos con drenaje extrahepático sin otros hallazgos asociados sobrevivió.

Tras el diagnóstico de ADV debemos por tanto valorar la anatomía del sistema portal fetal a partir de la segunda mitad de la gestación ya que no tiene gran dificultad, pero sí importancia pronóstica. Uno de los dos fetos con anomalía asociada del sistema porta falleció a las 12 horas de vida (caso 5).

\section{CONCLUSIÓN}

La ADV es por tanto una anomalía infrecuente del sistema venoso fetal, de difícil diagnóstico y prevalencia real desconocida pero con mal pronóstico en aquellos casos en que se asocia con otros marcadores y/o anomalías estructurales, que incluso pueden aparecer en la segunda mitad de la gestación, por lo que debe realizarse un seguimiento adecuado fundamentalmente mediante ultrasonidos. El incremento de grosor de la TN, a la que habitualmente se asocia la ADV, podría deberse a alteraciones hemodinámicas transitorias y no supone una clara indicación de estudio invasivo del cariotipo en ausencia de hallazgos estructurales y/o marcadores asociados. Su presencia es indicación de valoración morfológica detallada de toda la anatomía fetal y especialmente del corazón y sistema nervioso central que descarte anomalías asociadas y alteraciones del sistema portal.

\section{REFERENCIAS}

1. Mavrides E, Moscoso G, Carvalho JS, Campbell $S$, Thilaganathan B. The human ductus venosus between 13 and 17 weeks of gestation: histological and morphometric studies. Ultrasound Obstet Gynecol 2002;19:39-46.

2. Mavrides E, Moscoso G, Carvalho JS, Campbell S, Thilaganathan B. The anatomy of the umbilical, portal and hepatic venous systems in the human fetus at 14 -19 weeks of gestation. Ultrasound Obstet Gynecol 2001;18:598-604.

3. Kiserud T, Rasmussen S, Skulstad S. Blood flow and the degree of shunting through the ductus venosus in the human fetus. Am J Obstet Gynecol 2000;182:14753. 
4. Volpe $P$, Marasini M, Caruso G, Lituania M, Marzullo A, Volpe G. Prenatal diagnosis of ductus venosus agenesis and its association with cytogenetic/ congenital anomalies. Prenat Diagn 2002;22:9951000.

5. Contratti G, Banzi C, Ghi T, Perolo A, Pilu G, Visentin A. Abscence of the ductus venosus: report of 10 new cases and review of the literature. Ultrasound Obstet Gynecol 2001;18:605-9.

6. Matias A, Huggon I, Areias JC, Montenegro N, Nicolaides $\mathrm{KH}$. Cardiac defects in chromosomally normal fetuses with abnormal ductus venosus blood flow at 10-14 weeks. Ultrasound Obstet Gynecol 1999;14:307-10.
7. Yagel S, Kivilevitch Z, Cohen SM, Valsky DV, Messing B, Shen O, Achiron R. The fetal venous system, Part II: ultrasound evaluation of the fetus with congenital venous system malformation or developing circulatory compromise. Ultrasound Obstet Gynecol 2010;36:93111.

8. Shen O, Valsky DVV, Messing B, Cohen SM, Lipschuetz M, Yagel S. Shunt diameter in agenesis of ductus venosus with extrahepatic porto-systemic shunt impacts prognosis. Ultrasound Obstet Gynecol 2011;37:184-90.

9. Berg C, Kamil D, Geipel A, Kohl T, Knöpfle G, Hansmann M, Gembruch U. Absence of ductus venosus-importance of umbilical venous drainage site. Ultrasound Obstet Gynecol 2006;28:275-81. 